\title{
CHARACTERIZATIONS OF MULTINOMIAL DISTRIBUTIONS BASED ON CONDITIONAL DISTRIBUTIONS
}

\author{
KHOAN T. DINH', TRUC T. NGUYEN² and YINING WANG ${ }^{2}$ \\ 1U.S. Environmental Protection Agency \\ Washington, DC 20460 \\ 2Department of Mathematics and Statistics \\ Bowling Green State University \\ Bowling Green, $\mathrm{OH}$ 43403-0221
}

(Received March 8, 1994 and in revised form October 5, 1994)

\begin{abstract}
Several characterizations of the joint multinomial distribution of two discrete random vectors are derived assuming conditional multinomial distributions.
\end{abstract}

KEY WORDS AND PHRASES. Binomial distribution, joint distribution, conditional density, identically distributed, exchangeable distribution.

AMS 1992 SUBJECT CLASSIFICATION CODE. 62H05.

\section{INTRODUCTION.}

Suppose that the distributions of $X \mid Y=y$ and $Y \mid X=x$ both are given for every real values $x, y$, then the joint distribution of $X$ and $Y$ is tried to be reconstructed. Brucker [4], then Fraser and Streit [6], Castillo and Galambos [5] characterized a bivariate normal distribution given that $X \mid Y=y$ and $Y \mid X=x$ both have normal distribution under some given conditions. Bischoff and Fieger [3], then Hamedani [8] gave characterizations of multivariate normal distribution, Dinh and Nguyen [9] gave a characterization of matrix variate normal distribution. In the case $X$ and $Y$ are identically distributed, and $Y \mid X=x$ has a normal distribution of mean $a x+b$ and variance $\sigma^{2}$, Ahsanullah [1] showed that $|a|<1$ and $X$ and $Y$ have a joint bivariate normal distribution. In his paper Ahsanullah also proposed a conjecture for a multidimensional version of his result. Hamedani [7], then Arnold and Pourahmadi [2] gave counterexamples to this conjecture, and they also gave different characterizations for multivariate normal distribution based on conditional multivariate normality. Nguyen [10] gave a characterization for matrix variate normal distribution having identically distributed row vectors. In this note we consider the problem of characterization of multinomial distribution based on conditional multinomial distributions. In Section 2 a characterization of multinomial distribution is given based on two conditional multinomial distributions. In Section 3 a characterization of the joint multinomial of two identically distributed random vectors based on one conditional multinomial 
distribution is given. A conjecture similar to a conjecture proposed by Ahsanullah [1] is also raised and answered by a counterexample. Some supplementary conditions are added to this conjecture making it to be sufficient to characterize a multinomial distribution.

\section{THE FIRST CHARACTERIZATION.}

In this section we go to characterize a joint multinomial distribution of two discrete random vectors based on the conditional multinomial distribution of one vector given the other vector. A discrete random vector $X=\left(X_{1}, \ldots, X_{k}\right)^{\prime}$ is defined to have a multinomial $\left(n, p_{1}, \ldots, p_{k}\right)$ distribution if its density is given by

$$
p\left(x_{1}, \ldots, x_{k}\right)=\frac{n !}{\prod_{i=1}^{k} x_{i} !\left(n-\sum_{i=1}^{k} x_{i}\right) !} \prod_{i=1}^{k} p_{i}^{x_{i}}(1-P)^{n-\sum_{i=1}^{k} x_{1}}
$$

where $p_{i}>0, i=1, \ldots, k, \sum_{i=1}^{k} p_{i}<1,0 \leq \sum_{i=1}^{k} x_{i} \leq n, x_{i}$ nonnegative integer, $i=1, \ldots, k$, $P=\sum_{i=1}^{k} p_{i}$. Its moment generating function (m.g.f.) is given by

$$
M\left(s_{1}, \ldots, s_{k}\right)=\left(\sum_{i=1}^{k} p_{i} e^{s_{i}}+1-P\right)^{n},
$$

for all real numbers $s_{1}, \ldots, s_{k}$. It is clear that $M$ is a continuous function in $\left(s_{1}, \ldots, s_{k}\right)$.

THEOREM 2.1. Let $X=\left(X_{1}, \ldots, X_{m}\right)^{\prime}$ and $Y=\left(Y_{1}, \ldots, Y_{k}\right)^{\prime}$ be two discrete random vectors, whose components taking values on the set of nonnegative integers. Suppose $X \mid Y=y=\left(y_{1}, \ldots, y_{k}\right)$ has a multinomial $\left(n-\sum_{j=1}^{k} y_{j}, p_{1}, \ldots, p_{m}\right)$ distribution for all nonnegative integers $y_{1}, \ldots, y_{k}, \sum_{j=1}^{k} y_{j} \leq n$, and $\boldsymbol{Y} \mid \boldsymbol{X}=\boldsymbol{x}=\left(x_{1}, \ldots, x_{m}\right)$ has a multinomial $\left(n-\sum_{i=1}^{m} x_{i}, q_{1}, \ldots, q_{k}\right)$ distribution for all nonnegative integers $x_{1}, \ldots, x_{m}$, $\sum_{i=1}^{m} x_{i} \leq n$. Then $X$ and $Y$ have a joint multinomial distribution.

PROOF. From the conditional distribution of $X \mid Y=y$,

$$
P_{X, Y}(x, y)=\frac{\left(n-\sum_{j=1}^{k} y_{j}\right) !}{\prod_{i=1}^{m} x_{i} !\left(n-\sum_{i=1}^{m} x_{i}-\sum_{j=1}^{k} y_{j}\right) !} \prod_{i=1}^{k} p_{i}^{x_{i}}(1-P)^{n-\sum_{i=1}^{m} x_{i}-\sum_{j=1}^{k} y_{j}} P_{Y}(y),
$$

and from the conditional distribution of $Y \mid X=x$, 


$$
P_{X, Y}(x, y)=\frac{\left(n-\sum_{i=1}^{m} x_{i}\right) !}{\prod_{j=1}^{k} y_{j} !\left(n-\sum_{i=1}^{m} x_{i}-\sum_{j=1}^{k} y_{j}\right) !} \prod_{j=1}^{k} q_{j}^{y_{j}}(1-Q)^{n-\sum_{t=1}^{m} x_{i}-\sum_{j=1}^{k} y_{j}} P_{X}(x),
$$

where $Q=\sum_{i=1}^{k} q_{i}$. Equating (2.3) and (2.4), then simplifying,

$$
\frac{P_{X}(x) \prod_{i=1}^{m} x_{i} !\left(n-\sum_{i=1}^{m} x_{i}\right) !}{\prod_{i=1}^{m} p_{i}^{x_{i}}(1-Q)^{\sum_{i=1}^{m} x_{i}}(1-P)^{n-\sum_{i=1}^{m} x_{i}}}=\frac{p_{Y}(y) \prod_{j=1}^{k} y_{j} !\left(n-\sum_{j=1}^{k} y_{j}\right) !}{\prod_{j=1}^{k} q_{j}^{y_{j}}(1-P)^{\sum_{j=1}^{k} y_{j}}(1-Q)^{n-\sum_{j=1}^{k} y_{j}}},
$$

for all nonnegative integers $x_{1}, \ldots, x_{m}, y_{1}, \ldots, y_{k}, \sum_{i=1}^{m} x_{i}+\sum_{j=1}^{k} y_{j} \leq n$. The left side of (2.5) depends only on $x_{1}, \ldots, x_{m}$, meanwhile the right side of (2.5) depends only on $y_{1}, \ldots, y_{k}$, therefore their common value $K$ does not depend on $x$ and $y$. Hence,

$$
P_{X}(x)=\frac{K \prod_{i=1}^{m} p_{i}^{x_{i}}(1-Q)^{\sum_{i=1}^{m} x_{i}}(1-P)^{n-\sum_{i=1}^{m} x_{i}}}{\prod_{i=1}^{m} x_{i} !\left(n-\sum_{i=1}^{m} x_{i}\right) !}
$$

for all nonnegative integers $x_{1}, \ldots, x_{m}, \sum_{i=1}^{m} x_{i} \leq n$, and

$$
P Y(y)=\frac{K \prod_{j=1}^{k} q_{j}^{y_{j}}(1-P)^{\sum_{j=1}^{k} y_{j}}(1-Q){ }^{n-\sum_{j=1}^{k} y_{j}}}{\prod_{j=1}^{k} y_{j} !\left(n-\sum_{j=1}^{k} y_{j}\right) !},
$$

for all nonnegative integers $y_{1}, \ldots, y_{k}, \sum_{j=1}^{k} y_{j} \leq n$. To find $K$, sum up (2.6) on all possible values of $x$ or sum up (2.7) on all possible values of $y$, and using the fact that they are density functions,

$$
\sum_{x} P_{X}(x)=\frac{K}{n !} \sum_{x_{1}+\ldots+x_{m} \leq n} \frac{n !}{\left(\prod_{i=1}^{k} x_{i} !\right)\left(n-\sum_{i=1}^{k} x_{i}\right) !} \prod_{i=1}^{m}\left[p_{i}(1-Q)\right]^{x_{i}}(1-P)^{n-\sum_{i=1}^{k} x_{i}}
$$




$$
\begin{aligned}
& =\frac{K}{n !}\left[\sum_{i=1}^{m} p_{i}(1-Q)+1-P\right]^{n} \\
& =\frac{K}{n !}[1-P Q]^{n}=1 .
\end{aligned}
$$

Hence,

$$
K=\frac{n !}{[1-P Q]^{n}}
$$

Substitute $K$ in (2.6),

$$
P_{X}(x)=\frac{n !}{\prod_{i=1}^{m} x_{i} !\left(n-\sum_{i=1}^{m} x_{i}\right)} \prod_{i=1}^{m}\left[\frac{p_{i}(1-Q)}{1-P Q}\right]^{x_{i}}\left[\frac{1-P}{1-P(1-Q)}\right]^{n-\sum_{i=1}^{m} x_{i}}
$$

and then substitute (2.9) in (2.4),

$$
\begin{aligned}
P_{X, Y}(x, y)= & \frac{n !}{\prod_{i=1}^{m} x_{i} ! \prod_{j=1}^{k} y_{j} !\left(n-\sum_{i=1}^{m} x_{i}-\sum_{j=1}^{k} y_{j}\right) !} \prod_{i=1}^{m}\left[\frac{p_{i}(1-Q)}{1-P Q}\right]^{x_{i}} \\
& \cdot \prod_{j=1}^{k}\left[\frac{q_{j}(1-P)}{1-P Q}\right]^{y_{j}}\left[\frac{(1-P)(1-Q)}{1-P Q}\right]^{n-\sum_{i=1}^{m} x_{i}-\sum_{j=1}^{k} y_{j},}
\end{aligned}
$$

for all nonnegative integers $x_{1}, \ldots, x_{m}, y_{1}, \ldots, y_{k}, \sum_{i=1}^{m} x_{i}+\sum_{j=1}^{k} y_{j} \leq n$. Then $X$ and $Y$ have a joint multinomial distribution.

\section{THE SECOND CHARACTERIZATION.}

A characterization of the joint multinomial distribution of two identically distributed random vectors in this section is based on only one conditional multinomial distribution. It is trivial that if $X$ and $Y$ are random vectors whose components have values on the set of nonnegative integers and if $Y$ has a multinomial $\left(n, q_{1}, \ldots, q_{k}\right)$ distribution and $X \mid Y=y=\left(y_{1}, \ldots, y_{k}\right)$ has a multinomial $\left(n-\sum_{j=1}^{k} y_{j}, p_{2}, \ldots, p_{m}\right)$ distribution for all $y_{1}, \ldots, y_{k}$, $\sum_{j=1}^{k} y_{j} \leq n$, then the joint distribution of $X$ and $Y$ is multinomial with density given by

$$
\begin{aligned}
& P_{X, Y}(x, y)=\frac{n !}{\prod_{i=1}^{m} x_{i} ! \prod_{j=1}^{k} y_{j} !\left(n-\sum_{i=1}^{m} x_{i}-\sum_{j=1}^{k} y_{j}\right)}\left[\prod_{i=1}^{m}\left(p_{i}(1-Q)\right)\right. \\
& \cdot[(1-P)(1-Q)] \\
& n-\sum_{i=1}^{m} x_{i}-\sum_{j=1}^{k} y_{j}
\end{aligned}
$$

for all nonnegative integers $x_{1}, \ldots, x_{m}, y_{1}, \ldots, y_{n}$ such that $\sum_{i=1}^{m} x_{i}+\sum_{j=1}^{k} y_{j} \leq n$. 
If $X$ and $Y$ are identically distributed and if their joint distribution is a multinomial $\left(n, p_{1}, \ldots, p_{m}, p_{1}, \ldots, p_{m}\right)$ distribution, then $\sum_{i=1}^{m} p_{i}<\frac{1}{2}$ and the marginal distribution of $\mathbf{X}$ and $\boldsymbol{Y}$ is a multinomial $\left(n, p_{1}, \ldots, p_{m}\right)$ distribution. The conditional distribution of $\boldsymbol{X}$ given $Y=y=\left(y_{1}, \ldots, y_{m}\right)$ is a multinomial distribution with density given by

$$
P_{X \mid Y=y}(x \mid y)=\frac{\left(n-\sum_{j=1}^{m} y_{j}\right) !}{\prod_{i=1}^{m} x_{i} !\left(n-\sum_{i=1}^{m} x_{i}-\sum_{j=1}^{m} y_{j}\right) !}\left[\prod_{i=1}^{m}\left(\frac{p_{i}}{1-P}\right)^{x_{i}}\right]\left[\frac{1-2 P}{1-P}\right]^{n-\sum_{j=1}^{m} y_{j}-\sum_{i=1}^{m} x_{i},}
$$

for all nonnegative integers $x_{1}, \ldots, x_{m}$ such that $\sum_{i=1}^{m} x_{i} \leq n-\sum_{j=1}^{m} y_{j}$.

THEOREM 3.1. Let $X=\left(X_{1}, \ldots, X_{m}\right)^{\prime}$ and $Y=\left(Y_{1}, \ldots, Y_{m}\right)^{\prime}$ be two identically distributed discrete random vectors whose components have values on the set nonnegative integers. Suppose $X \mid Y=y=\left(y_{1}, \ldots, y_{m}\right)^{\prime}$ has a multinomial $\left(n-\sum_{j=1}^{m} y_{j}, p_{1}, \ldots, p_{k}\right)$ distribution for all nonnegative integers $y_{1}, \ldots, y_{m}, \sum_{j=1}^{m} y_{j} \leq n$, then $X$ and $\boldsymbol{Y}$ have a joint multinomial distribution.

PROOF. Since $X \mid Y=y=\left(y_{1}, \ldots, y_{m}\right)$ has a multinomial $\left(n-\sum_{j=1}^{m} y j, p_{1}, \ldots, p_{k}\right)$ distribution, the joint distribution of $X$ and $Y$ is given by

$$
P_{X, Y}(x, y)=\frac{\left(n-\sum_{j=1}^{m} y_{j}\right) !}{\prod_{i=1}^{m} x_{i} !\left(n-\sum_{i=1}^{m} x_{i}-\sum_{j=1}^{m} y_{j}\right) !}\left(\prod_{i=1}^{m} p_{i}^{x_{i}}(1-P)^{n-\sum_{i=1}^{m} x_{i}-\sum_{j=1}^{m} y_{j}} P(y),\right.
$$

where $P$ is the marginal density function of $X$ and $Y, x_{1}, \ldots, x_{m}, y_{1}, \ldots, y_{m}$ are nonnegative integers, $\sum_{i=1}^{m} x_{i}+\sum_{j=1}^{m} y_{j} \leq n$. Hence, the range of each component is from 0 to $n$, and the m.g.f. $M\left(s_{1}, \ldots, s_{m}\right)$ of $X$ and $Y$ is a continuous function in $R^{m}, M>0$ for all $\left(s_{1}, \ldots, s_{m}\right)$ of $R^{m}$. The joint m.g.f. of $X$ and $Y$ is given by

$$
\begin{aligned}
M_{X, Y}(s, t) & =E\left[e^{s^{\prime} X+t^{\prime} Y}\right]=E\left[e^{t^{\prime} Y} E\left[e^{s^{\prime} X} \mid Y\right]\right] \\
& =E\left[e^{t^{\prime} Y}\left(\sum_{i=1}^{m} p_{i} e^{s_{i}}+1-P\right)^{n-\sum_{i=1}^{m} Y_{i}}\right] \\
& =\left(\sum_{i=1}^{m} p_{i} e^{s_{i}}+1-P\right)^{n} E\left[e^{\left[\left(-1 \ln \left(\sum_{i=1}^{m} p_{i} e^{s_{t}}+1-P\right)\right)^{\prime} Y\right]}\right.
\end{aligned}
$$




$$
=\left(\sum_{i=1}^{m} p_{i} e^{s_{l}}+1-P\right)^{n} M\left(t-1 \ln \left(\sum_{i=1}^{m} p_{i} e^{s_{l}}+1-P\right)\right) \text {, }
$$

where $\mathbf{1}=(1, \ldots, 1)^{\prime}$ of $R^{m}$, for all $s=\left(s_{1}, \ldots, s_{m}\right)^{\prime}, t=\left(t_{1}, \ldots, t_{m}\right)^{\prime}$ of $R^{m}$. Hence,

$$
M(s)=\left(\sum_{i=1}^{m} p_{l} e^{s_{l}}+1-P\right)^{n} M\left(-1 \ln \left(\sum_{i=1}^{m} p_{l} e^{s_{l}+1-P}\right)\right)
$$

for all $s \in R^{m}$.

Let $M_{1}$ and $M_{2}$ be two m.g.f. solutions of (3.4). Set $\frac{M_{1}(s)}{M_{2}(s)}=h(s)$. Then $h(s)$ is continuous on $R^{m}$ and $h(0)=1$. From

$$
M_{1}(s)=\left(\sum_{i=1}^{m} p_{l} e^{s_{l}}+1-\sum_{i=1}^{m} p_{i}\right)^{n} M\left(-1 \ln \left(\sum_{i=1}^{m} p_{i} e^{s_{l}}+1-\sum_{i=1}^{m} p_{i}\right)\right)
$$

and

$$
\begin{gathered}
M_{2}(\mathrm{~s})=\left(\sum_{i=1}^{m} p_{i} e^{s_{l}}+1-\sum_{i=1}^{m} p_{i}\right)^{n} M_{2}\left(-1 \ln \left(\sum_{i=1}^{m} p_{i} e^{s_{i}}+1-\sum_{i=1}^{m} p_{i}\right)\right), \\
h(s)=h\left(1 \ln \left(\frac{1}{\sum_{i=1}^{m} p_{i} e^{s_{i}}+1-P}\right)\right)
\end{gathered}
$$

for all $s$ of $R^{m}$.

Iterating the right side of (3.7) using (3.7) itself,

$$
h(s)=h\left(1 \ln A_{1}\right)=h\left(1 \ln A_{2}\right)=\ldots=h\left(1 \ln A_{\ell}\right)=\ldots,
$$

where the sequence $\left\{A_{\ell}\right\}, \ell=1,2,3, \ldots$, is defined recursively by

$$
A_{1}=\frac{1}{\sum_{i=1}^{m} p_{i} e^{s_{i}}+1-P}, \quad A_{\ell}=\frac{1}{P A_{\ell-1}+1-P} \text { for all } \ell=2,3, \ldots .
$$

It is trivial to show that if $A_{1} \geq 1$, then $A_{1} \geq A_{3} \geq A_{5} \geq \ldots \geq 1$ and $A_{2} \leq A_{4} \leq \ldots \leq 1$, and if $A_{1} \leq 1, A_{1} \leq A_{3} \leq A_{5} \leq \ldots \leq 1$ and $A_{2} \geq A_{4} \geq \ldots \geq 1$. The two sequences

$\left\{A_{2 \ell-1}\right\}$ and $\left\{A_{2 \ell}\right\}$ are convergent and both of them converge to 1 . Hence, by the fact that the function $h$ is continuous on $R^{m}$ and the function $\ell n$ is continuous on $(0, \infty)$,

$$
h(s)=h\left(1 \ln \left(\lim _{n \rightarrow \infty} A_{n}\right)\right)=h(1.0)=h(0)=1 .
$$

Therefore the equation (3.4) has a unique solution. By (3.2) this solution is the m.g.f. of a multinomial distribution, and $Y$ has a multinomial distribution. By the result (3.1), the joint distribution of $\boldsymbol{X}$ and $\boldsymbol{Y}$ is multinomial.

The following question will be studied regarding multinomial distribution. If $X_{1}, \ldots, X_{k}$ are identically distributed discrete random variables having values on the set nonnegative integers, where $k \geq 3$ and if $X_{1} \mid X_{2}=x_{2}, \ldots, X_{k}=x_{k}$ has a binomial $\left(n-\sum_{i=2}^{k} x_{i}, p\right)$ distribution $0<p<1$ for all $x_{2}, \ldots, x_{k}$ nonnegative integers, $\sum_{i=2}^{k} x_{i} \leq n$, then does it imply that $X_{1}, \ldots, X_{k}$ have a joint multinomial distribution? The answer for this question is given by the following counterexample.

EXAMPLE 3.1. Let $X_{1}, X_{2}, X_{3}$ be three discrete random variables having a joint density function 


$$
\begin{gathered}
P(0,0,0)=P(2,0,0) \doteq P(0,2,0)=P(0,0,2)=1 / 9 \\
P(1,0,0)=P(0,1,1)=2 / 9 \\
P(0,0,1)=P(1,0,1)=P(0,1,0)=P(1,1,0)=1 / 36 .
\end{gathered}
$$

Then it is trivial that $X_{1}, X_{2}, X_{3}$ are identically distributed with density function $P(0)=11 / 18, P(1)=5 / 18, P(2)=2 / 18$,

and this is not a binomial $(2, p)$ distribution, since there does not exist any $p$ for a binomial $(2, p)$ distribution to fit to this distribution. This fact shows that the joint distribution of $X_{1}, X_{2}, X_{3}$ is not a trinomial distribution, meanwhile, it is easy to check that $X_{1} \mid X_{2}=x_{2}, X_{3}=x_{3}$ has a binomial $\left(2-x_{2}-x_{3}, \frac{1}{2}\right)$ distribution for all $x_{2}, x_{3}, x_{2}+x_{3} \leq 2$.

In addition to the given conditions of the above question, if $X_{1}, \ldots, X_{k}$ are supposed to have an exchangeable distribution in $x_{1}, \ldots, x_{k}$, we go to show that the joint distribution of $X_{1}, \ldots, X_{k}$ is a multinomial distribution.

If $k=2$, since $X_{1}$ and $X_{2}$ are identically distributed and $X_{1} \mid X_{2}=x_{2}$ has a binomial $\left(n-x_{2}, p\right)$ distribution, by Theorem 3.1, $X_{1}$ and $X_{2}$ have a joint trinomial distribution. For $k>2$, the proof follows by mathematical induction. For some positive integer $k \geq 2$, suppose that for any $m=2, \ldots, k$, if $X_{1} \mid X_{2}=x_{2}, \ldots, X_{m}=x_{m}$ has a binomial $\left(n-\sum_{i=2}^{m} x_{i}, p\right)$ distribution, then the joint distribution of $\left(X_{1}, \ldots, X_{m}\right)$ is a multinomial distribution, we go to show that it is also true if the number of random variables is $k+1$.

From $X_{1} \mid X_{2}=x_{2}, \ldots, X_{k}=x_{k}, X_{k+1}=x_{k+1}$ has a binomial $\left(n-\sum_{i=2}^{k+1} x_{i}, p\right)$ distribution, then

$$
\begin{aligned}
P\left(x_{1} \mid x_{2}, \ldots, x_{k}, x_{k+1}\right) & =\frac{P\left(X_{1}=x_{1}, X_{2}=x_{2}, \ldots, X_{k}=x_{k}, X_{k+1}=x_{k+1}\right)}{P\left(X_{2}=x_{2}, \ldots, X_{k+1}=x_{k+1}\right)} \\
& =\frac{P\left(X_{1}=x_{1}, \ldots, X_{k}=x_{k}, X_{k+1}-x_{k+1}\right) / P\left(X_{3}=x_{3}, \ldots, X_{k+1}=x_{k+1}\right)}{P\left(X_{2}=x_{2}, \ldots, X_{k}=x_{k}, X_{k+1}=x_{k+1}\right) / P\left(X_{3}=x_{3}, \ldots, X_{k+1}=x_{k+1}\right)} .
\end{aligned}
$$

Set $Y_{1}=X_{1} \mid X_{3}=x_{3}, \ldots, X_{k+1}=x_{k+1}$, and $Y_{2}=X_{2} \mid X_{3}=x_{3}, \ldots, X_{k+1}=x_{k+1}$. Then $P\left(Y_{1}=x_{1} \mid Y_{2}=x_{2}\right)=P\left(x_{1} \mid x_{2}, \ldots, x_{k+1}\right)$ and $Y_{1} \mid Y_{2}=x_{2}$ has a binomial $\left(n-\sum_{i=2}^{k} x_{i}, p\right)$ distribution, and $Y_{1}$ and $Y_{2}$ are identically distributed since $X_{1}, \ldots, X_{k+1}$ have a joint exchangeable distribution in $x_{1}, \ldots, x_{k+1}$. By Theorem 3.1, $Y_{1}$ and $Y_{2}$ have a binomial $\left(n-\sum_{i=3}^{k+1} x_{i}, q\right)$ distribution for some $0<q<1$. From $Y_{2}=X_{2} \mid X_{3}=x_{3}, \ldots, X_{k+1}=x_{k+1}$ has a binomial $\left(n-\sum_{i=3}^{k+1} x_{i}, q\right)$ distribution and by induction hypothesis, $X_{2}, \ldots, X_{k+1}$ have a joint multinomial distribution. Hence, $X_{1}, \ldots, X_{k+1}$ have a joint multinomial distribution. By mathematical induction principle, this result of joint multinomial of $X_{1}, \ldots, X_{k}$ is true for all $k \geq 2$. Therefore the following result is proved.

THEOREM 3.2. Let $X_{1}, \ldots, X_{k}$ be identically distributed random variables having values on the set of nonnegative integers. Suppose that their joint density is 
exchangeable in $x_{1}, \ldots, x_{k}$, and $X_{1} \mid X_{2}=x_{2}, \ldots, X_{k}=x_{k}$ has a binomial $\left(n-\sum_{i=2}^{k} x_{l}, p\right)$ distribution, then $X_{1}, \ldots, X_{k}$ have a joint multinomial distribution.

Theorem 3.2 can be generalized to Theorem 3.3. below in the case of identically distributed random vectors by using the result of Theorem 3.1 and a similar proof for Theorem 3.2.

THEOREM 3.3. Let $X_{1}, \ldots, X_{k}$ be identically distributed $m \times 1$ random vectors whose components have values on the set of nonnegative integers. Suppose that the joint density of $X_{1}, \ldots, X_{k}$ is exchangeable in $x_{1}, \ldots, x_{k}$ and $X_{1} \mid X_{2}=x_{2}, \ldots, X_{k}=x_{k}$ has a multinomial

$\left(n-\sum_{i=2}^{k} \sum_{j=1}^{m} x_{i j}, p_{1}\right)$ distribution, where $x_{i}=\left(x_{l, 1}, \ldots, x_{l, m}\right)^{\prime}, i=2, \ldots, k, p_{1}=\left(p_{1,1}, \ldots, p_{1, m}\right)^{\prime}$, then $X_{1}, \ldots, X_{k}$ have a joint multinomial distribution.

ACKNOWLEDGEMENT. The authors would like to thank the referee for helpful suggestions and comments.

\section{REFERENCES}

[1] Ahsanullah, M. (1985). Some characterizations of the bivariate normal distribution. Metrika 32, 215-218.

[2] Arnold, B. C. and Pourahmadi, M. (1988). Conditional characterizations of multivariate distributions. Metrika 35, 99-108.

[3] Bischoff, W. and Fieger, W. (1991). Characterization of the multivariate normal distribution by conditional normal distribution. Metrika 38, 239-248.

[4] Brucker, J. 91979). A note on the bivariate normal distribution. Commun. Statist. Theor. Meth. A8(2), 175-177.

[5] Castillo, E. and Galambos, J. (1989). Conditional distributions and the bivariate normal distribution. Metrika 36, 209-214.

[6] Fraser, D. A. S. and Streit, F. (1980). A further note on the bivariate normal distribution. Commun. Statist. Theor. Meth., A9(10), 1097-1099.

[7] Hamedani, G. G. (1988). On two recent characterizations of multivariate normal distribution. Metrika 35, 41-47.

[8] Hamedani, G. G. (1992). Bivariate and multivariate normal characterization. A brief survey. Commun. Statist. Theor. Methods 21(9), 2665-2688.

[9] Dinh, K. T. and Nguyen, T. T. (1994). A characterization of matrix variate normal distribution. Inter. Jour. of Math. and Math. Science. 17(2), 341-346.

[10] Nguyen, T. T. (1993). A note on matrix variate normal distribution. Technical Report. Department of Mathematics and Statistics, Bowling Green State University, Bowling Green, $\mathrm{OH}$ 43403-0221. 


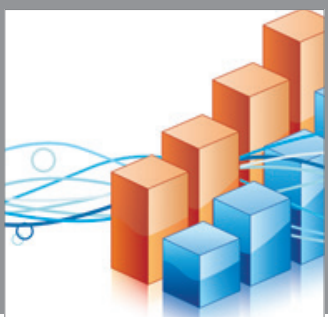

Advances in

Operations Research

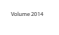

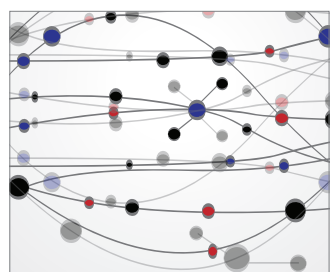

\section{The Scientific} World Journal
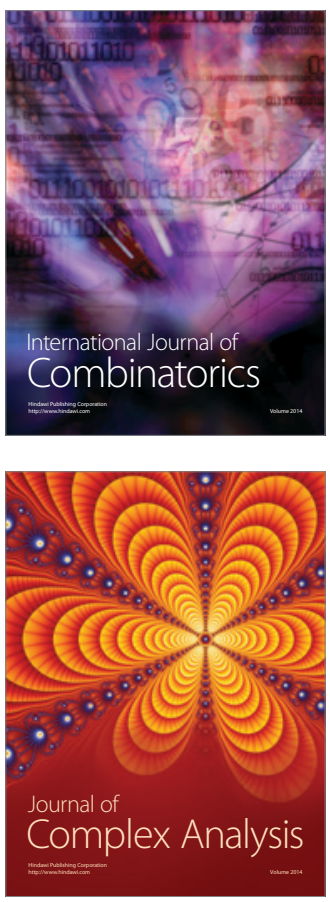

International Journal of

Mathematics and

Mathematical

Sciences
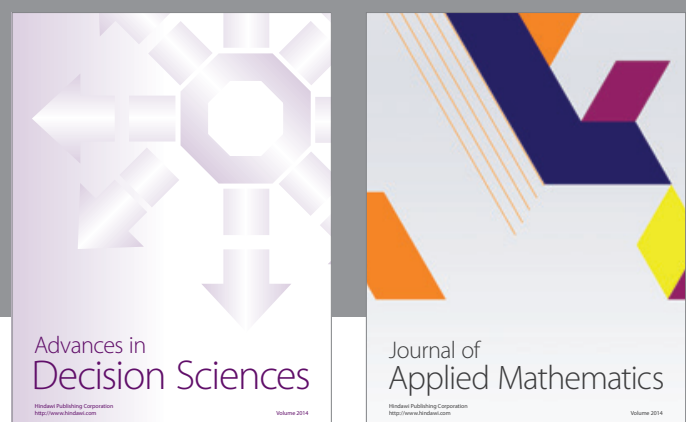

Journal of

Applied Mathematics
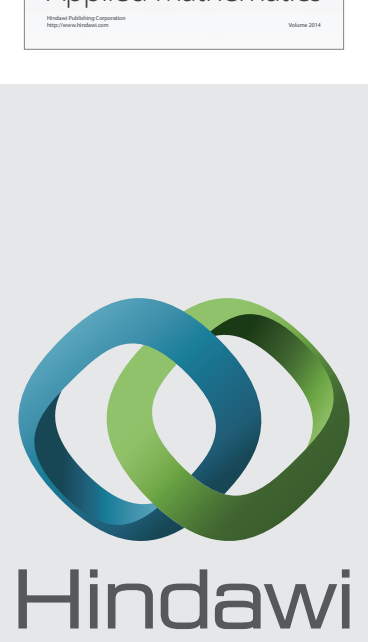

Submit your manuscripts at http://www.hindawi.com
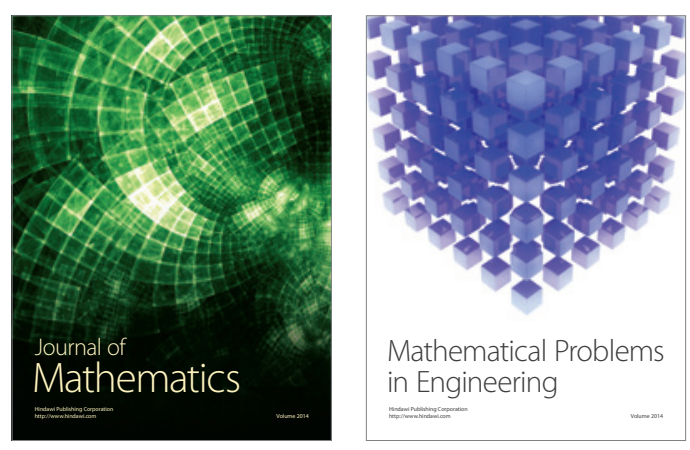

Mathematical Problems in Engineering
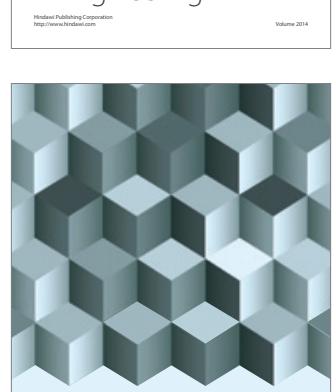

Journal of

Function Spaces
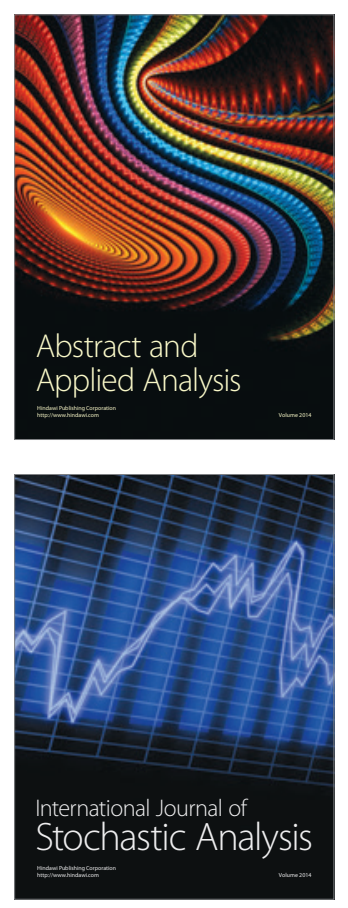

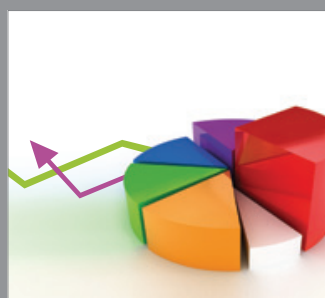

ournal of

Probability and Statistics

Promensencen
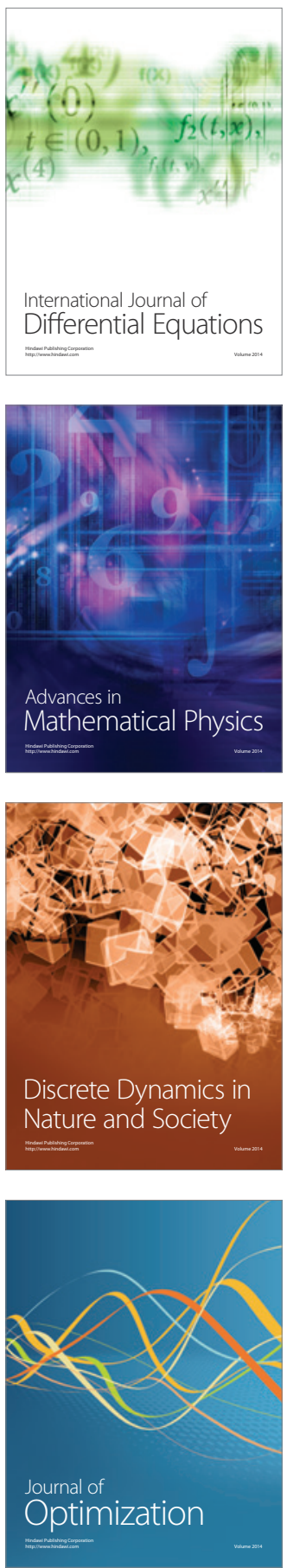